\title{
Au cœur de l'album, le conte Cœur de bois de Henri Meunier et Régis Lejonc
}

\section{Christiane Connan-Pintado}

\section{(2) OpenEdition}

\section{Journals}

Édition électronique

URL : https://journals.openedition.org/edl/1560

DOI : $10.4000 /$ edl. 1560

ISSN : 2296-5084

Éditeur

Université de Lausanne

\section{Édition imprimée}

Date de publication : 15 septembre 2019

Pagination : 21-40

ISBN : 978-2-940331-71-0

ISSN : 0014-2026

\section{Référence électronique}

Christiane Connan-Pintado, « Au cœur de l'album, le conte Cœur de bois de Henri Meunier et Régis Lejonc ", Études de lettres [En ligne], 310 | 2019, mis en ligne le 15 septembre 2021, consulté le 22 septembre 2021. URL : http://journals.openedition.org/edl/1560 ; DOI : https://doi.org/10.4000/edl. 1560

Ce document a été généré automatiquement le 22 septembre 2021.

(c) Études de lettres 


\title{
Au cœur de l'album, le conte Cour de bois de Henri Meunier et Régis Lejonc
}

\author{
Christiane Connan-Pintado
}

Au milieu du chemin de notre vie je me retrouvai par une forêt obscure

car la voie droite était perdue. Ah dire ce qu'elle était est chose dure

cette forêt féroce et âpre et forte qui ranime la peur dans la pensée !1

1 Pièce incontournable du patrimoine, le conte $\mathrm{du}$ « Petit Chaperon rouge » occupe une place privilégiée dans l'imaginaire collectif et dans les formes les plus variées de la représentation. Au point qu'Anne-Marie Garat commente son emprise en ces termes :

Face à la production moderne qui ne manque pourtant pas de monstres, ce conte-là ne cède pas d'un pouce. Il ne s'use pas comme d'autres [...]. Il résiste au temps, par sa vitalité remarquable, son pouvoir étrange. [II] reste saignant dans notre mémoire, intact dans sa jeune nouveauté, paré de sa séduction et de son épouvante premières ${ }^{2}$.

Ce conte «saignant" s'impose donc aussi dans l'univers éditorial: auteurs et illustrateurs ne cessent de remettre sur le métier sa trame ténue pour la tisser autrement; quant aux critiques, ils en déplient le feuilleté pour soupeser et faire signifier chaque mot, chaque image, chaque ellipse ${ }^{3}$.

En l'espèce, "Le Petit Chaperon rouge " inspire deux auteurs habitués à créer en tandem des albums pour la jeunesse. Familier de l'espace de jeu offert par les contes, Henri Meunier a publié plusieurs variations parodiques ${ }^{4}$. Quant à Régis Lejonc, il a déjà illustré un Petit Chaperon rouge 5 . Paru à Genève en 2016, aux Éditions Notari, l'album iconotextuel intitulé Couur de bois ${ }^{6}$ retient notre attention à plusieurs titres, car tout en s'inscrivant dans un contexte contemporain, il jongle habilement avec les sources du conte. À la fois ludique et grave, il se place sous le signe de l'hybridité pour exploiter les ressources du mariage entre conte et album et pour développer un propos aux vertus 
curatives. Il traite en effet de résilience et s'inscrit dans le courant littéraire contemporain qui vise à "réparer le monde", pour reprendre le titre de l'ouvrage d'Alexandre Gefen?.

4 Le récit peut se résumer en quelques mots : une jeune femme rend visite à un vieillard et l'accompagne pour une promenade en forêt. Mais cette trame prend une tout autre signification lorsque l'on reconnaît dans ce couple la fillette en rouge devenue adulte et le loup désormais infirme. Ce qui se joue alors entre les deux personnages tient à la fois $\mathrm{du}$ règlement de comptes et de la réparation. Au cœur de la forêt, au creux du conte, les atouts graphiques et plastiques de l'album concourent à mettre à nu le noyau dur du drame qui lie à jamais les deux personnages.

On s'intéressera successivement aux traces du conte qui affleurent ici et là, aux moyens de l'album pour mettre en valeur la recréation du conte et à la portée idéologique complexe de cette transcréation.

\section{Le conte sous le palimpseste}

Si le lecteur ne pense pas forcément à l'univers du conte lorsqu'il découvre l'ouvrage, les indices de son empreinte le frappent rétrospectivement lors de la relecture. Que l'hérö̈ne se prénomme Aurore convoque d'emblée - c'est le premier mot du texte - le conte de « La Belle au bois dormant » dans la version de Disney, qui attribue à la Belle le prénom de sa fille chez Perrault. Qu'elle se regarde dans le miroir en s'exclamant « La plus belle, c'est moi. Et merde à Blanche-Neige ! » (9) l'inscrit dans un réseau de contes centrés sur de jeunes femmes que leur beauté distingue, pour leur malheur. Les actions anodines qui s'enchaînent dans la première partie de l'album : se préparer, acheter un gâteau, prendre la route et rêvasser au volant, attestent un "épanchement du conte " ${ }^{8}$ qui s'impose en deuxième lecture. En effet, celle que le loup appellera plus loin « fillette » «fredonn[e] une comptine » en pensant à ses propres filles, autres fillettes dont l'auteur fait rimer les désignations : « la pauvrette », « la cadette ». Henri Meunier tisse habilement trivialité du quotidien et échos au conte (12-13) : plus loin, on apprend que le gâteau est une galette, comme il se doit en janvier, mais aussi comme dans le texte de Perrault; lors de la promenade, clin d'œil aux versions populaires ${ }^{9}$, Aurore interroge sur la direction à prendre : "Chemin des Aiguilles ou Chemin des Épingles ?» (26). Quant au drame d'autrefois, il est rappelé dans les mêmes termes par les deux protagonistes : «moi qui, naguère, vous ai dévorée toute crue » $(29)$; «Vous m'avez dévorée hier». De plus, Aurore mentionne les «crocs» (30) du loup, dans une métonymie qui identifie l'agresseur à son arme.

7 Ce tissage intertextuel arrime le récit contemporain au conte source, alors que de subtiles différences invitent à l'en distinguer. Parmi les éléments de décalage, l'âge des personnages - Aurore a « la quarantaine généreuse », le loup est un «vieillard » (9) classe cette nouvelle histoire dans la catégorie de la "continuation" qui vise, selon Gérard Genette ${ }^{10}$, à procurer à un texte l'achèvement qui lui manquait. Richard SaintGelais précise l'analyse :

Donner à un récit un prolongement, c'est remettre en question les limites que se fixait l'œuvre originale. Un tel geste ne saurait être innocent $[. . .]^{11}$.

8 En effet, le procédé ouvre le champ à un questionnement qui se fonde " d'abord et avant tout sur la dynamique du récit avec ce qu'elle implique d"“ aspiration " vers le “qu'arrivera-t-il ensuite?" qui implique bien davantage qu'une curiosité 
chronologique ${\aleph^{12}}$. Il conviendra de s'interroger sur la portée idéologique de ce prolongement.

9 Confronter les étapes du nouveau récit à celles de ses sources permet de mettre en évidence les déplacements opérés. Alors que dans le conte de Perrault, comme dans le conte populaire ${ }^{13}$, l'héroïne est une enfant placée entre deux mères ${ }^{14}$ reliées par un jeu d'échange de parure et de nourriture (chaperon confectionné par l'une, galette par l'autre), celle de l'album est une femme au mitan de sa vie, elle-même mère et qui, de sa propre initiative, se donne pour mission de porter une part de galette à une vieille connaissance en souffrance, au fond du bois. Quant au loup, c'est sans la moindre ruse qu'il occupe cette fois pleinement la situation de la grand-mère dans sa maison de la forêt, vieux, malade, alité, assisté, isolé dans une complète déréliction. Non seulement le rapport de forces s'est objectivement inversé, mais c'est en connaissance de cause que son ancienne victime lui rend visite.

Dans la mesure où la réécriture s'inscrit dans le cadre d'un album iconotextuel, elle unit deux codes sémiotiques dont les discours croisés s'enrichissent de cette association. Les deux artistes travaillent fréquemment ensemble et leur collaboration se montre attentive aux effets de sens procurés par le frottement du texte et des images, afin de mettre au jour les arrière-plans du récit.

\section{Les révélations de l'image}

Tout fait sens dans un album, son format, son texte, ses images et leur interrelation. La première de couverture de cœur de bois intrique les trois composantes de façon apéritive. Le format très allongé $(30,5 \times 22,5 \mathrm{~cm})$ se place au service du décor représenté, de tonalité sombre : une forêt hivernale, dans laquelle s'enfonce en bas de page, au centre, un étroit chemin où s'engage une petite voiture rouge, que le regard ne découvre que dans un second temps. Elle est cernée par les fûts des arbres démesurément étirés, serrés comme des barreaux, ouvrant seulement à leur cime une clairière de ciel pâle, au centre de laquelle s'inscrivent en lettres noires les noms des auteurs et le titre de l'album (fig. 1). Certes, la conjonction de la forêt profonde et de la tache rouge active la convocation subliminale du conte du « Petit Chaperon rouge ». De plus, si cela échappe au premier regard, on découvre par la suite que, sur la plaque minéralogique de la voiture, se laissent déchiffrer des signes qui réfèrent aux sources de la réécriture : on reconnaît dans « $\mathrm{CP}$ AT 333 » les initiales de Perrault et le codage du conte pour la classification internationale d'Aarne et Thompson (fig. 2). Le jeu des indices iconographiques est donc lancé d'emblée, à charge au lecteur détective de les identifier.

$<$ Image en attente de droits>

Fig. 1 - H. Meunier et R. Lejonc, Cœeur de bois. @ Éditions Notari, 2016.

$<$ Image en attente de droits>

Fig. 2 - H. Meunier et R. Lejonc, Cœur de bois. @ Éditions Notari, 2016.

12 Texte et images s'accordent pour situer la diégèse dans un contexte contemporain anodin. Sans texte, la première double page (6-7) plante le cadre : un village avec son clocher, une poignée de maisons éparpillées sous un vaste ciel d'hiver, des champs enneigés, des arbres dénudés. Quand on pénètre dans ce paisible décor, c'est pour voir la boulangerie-pâtisserie devant laquelle se gare la petite voiture (10-11). Le projet 
d'Aurore a été annoncé à la page précédente : elle part faire « une balade en forêt avec un vieillard impotent». Au début du trajet, le narrateur donne accès aux préoccupations d'une mère de famille ordinaire qui songe au repas du soir, à la santé de ses enfants, à la grève des bus scolaires, aux vacances prochaines. Puis, dans un ralenti bucolique, il fait goûter au lecteur les sensations d'Aurore dans la forêt, une fois descendue de voiture, souriant sous l'effet de l'air vif, attentive "à l'activité de la faune engourdie", aux "légers craquements sous ses pas». Loin des terres habitées et des routes balisées, son avancée résolue sur le "petit sentier pédestre de la forêt domaniale » la mène jusqu'à " un pavillon de chasse délabré. Le fantôme d'une superbe passée» (16-17). Les deux doubles pages suivantes s'attardent sur les gestes d'Aurore dans la maison : aérer et nettoyer une cuisine malodorante et en désordre, préparer un plateau pour le thé, couper la galette, placer la fève et la couronne dans la part du vieillard « assoupi dans son fauteuil ». Le " vieillard » est ainsi désigné par le texte dans tout l'album (avec neuf occurrences du terme et deux pour "vieux»), lui dont l'apparition est retardée jusqu'au moment où la tourne de page le dévoile enfin, en fausse page, quand l'image s'écarte du texte pour jouer sa partition en solo (fig. 3). Il s'agit en effet du loup, un très vieux loup, à la face et au poitrail grisâtres et ridés, voûté, avachi dans un fauteuil : sa silhouette occupe frontalement toute la page et son regard se plante dans celui du lecteur. Un loup à la " superbe passée » mais qui, d'après le bref échange qui chapeaute l'image, tente de rester bravache : " Vous avez faim ?", demanda-t-elle? "Toujours", répondit-il» (22). Si la révélation de la nature du personnage agresse le lecteur surpris, c'est parce que «L'animalité permet également de suggérer la sexualité, sans l'énoncer explicitement ", comme l'écrit Jeanne Bloch à propos des contes qui illustrent le motif du fiancé animal. Elle y voit en effet une "métaphore des rapports entre hommes et femmes. L'animalité est aussi porteuse d'une importante dimension sexuelle. [...] Ces contes se présentent alors comme autant de variations sur le thème de la belle et la bête, avec tout ce que cela peut impliquer en termes de symboles de douceur et d'horreur, de féminin et de masculin $»^{15}$.

$<$ Image en attente de droits>

Fig. 3 - H. Meunier et R. Lejonc, Cœur de bois. @ Éditions Notari, 2016.

13 La suite de la visite se centre sur la promenade en forêt et le dialogue des deux personnages. L'image accuse le contraste entre l'énergie, la verticalité, la grâce d'Aurore et le délabrement du loup, qui a perdu toute autonomie. Le texte mentionne "son corps maigrelet", "ses jambes flageolantes", et Aurore se dit "qu'il ne pèse guère plus qu'un fagot de bois mort ». L'image complète le tableau de cette déchéance : en essayant de se lever seul, il renverse la table basse, casse son assiette, fait rouler sa couronne à terre et elle doit jeter le corps inerte sur son épaule afin de l'installer sur la chaise roulante (24-25). Ils s'enfoncent alors dans la forêt, elle poussant la chaise, lui corps tassé, tête enfouie dans une chapka, jambes (le texte n'use que de termes humains pour le décrire) sous un plaid rouge. La couleur rouge, emblème de l'enfant du conte, enveloppe à présent le loup, comme le souligne le gros plan qui cadre le fauteuil à roulettes et le maigre bras-patte velu crispé sur l'accoudoir (29). Il demande vite à rentrer, car il a froid, et se lamente sur sa situation, lui qui vit reclus, malade, abandonné de tous, sauf d'Aurore, justement : «Je n'ai que vous, fillette ». Ce troublant renversement des positions victimaires n'est pas celui qui fait florès dans les productions parodiques pour la jeunesse depuis les années 1970, car elles se contentent d'inverser les rôles pour divertir les lecteurs, au risque de construire de nouveaux 
stéréotypes de loups végétariens face à des chaperons délurés. Le propos de notre album est d'un autre ordre.

L'image réserve une autre révélation, à travers le choix de Régis Lejonc pour représenter Aurore. Le personnage paraît d'entrée, belle au miroir de dos, alors qu'elle se prépare, "forc[e] le trait de khôl sur ses yeux ", " coinc[e] sous son béret quelques mèches » et « dépos[e] deux gouttes de parfum à la naissance de son cou » car « même pour une balade dans la forêt avec un vieillard impotent, elle voulait être irrésistible " (8-9). Irrésistible en effet, en icône médiatique, voire en mythologie barthésienne, Aurore a les traits de Brigitte Bardot en 1968, lorsqu'elle chante "Bonnie \& Clyde " avec Serge Gainsbourg, en arborant la même coupe de cheveux et le même béret que Faye Dunaway dans le film d'Arthur Penn, l'année précédente. Ce visage se devine dans le reflet du miroir, car Aurore n'offre au lecteur que les courbes de sa silhouette, vue de dos. Le choix de Bardot pour ce personnage de quadragénaire qui a "une allure de jeune femme et un teint de gamine » (9) atteste la volonté de l'illustrateur de souligner la séduction sensuelle du personnage et d'aborder le conte du " Petit Chaperon rouge " sans en masquer les enjeux. Perrault ménageait déjà la dimension crossover $^{16} \mathrm{~d}^{\prime}$ un conte dans lequel est relatée l'aventure d'une "petite fille de village " alors que sa moralité s'adresse aux "jeunes filles/Belles, bien faites et gentilles" qui ne se méfient pas suffisamment des «loups doucereux/De tous les loups [...] les plus dangereux $»^{17}$. Régis Lejonc avait exploré ce double registre dans sa précédente illustration d'un Petit Chaperon rouge ${ }^{18}$ inspiré des versions populaires : au fil des pages, on y voyait grandir la fillette, soudain adolescente au moment de se déshabiller pour rejoindre le loup dans le lit, puis jeune femme lors de sa fuite, nue, dans la nature. Une telle évolution ne faisait pas mystère du sens à donner au péril d'être dévorée. L'iconographie de la Renaissance et du XVII ${ }^{e}$ siècle regorge de Vénus au miroir fameuses et un autre album pour la jeunesse s'en est souvenu en 1991 pour une recréation sulfureuse du «Petit Chaperon rouge » : dans Mina je t'aime $e^{19}$, la Lolita rousse qui s'apprête à partir chez sa grand-mère se contemple dans un miroir, elle aussi montrée de dos, vêtue d'une minirobe rouge qui laisse apercevoir sa culotte.

\section{Les voies de la résilience}

En fin d'album, le «visage impassible » d'Aurore-Bardot ${ }^{20}$, «mine songeuse » (30), s'affiche en belle page (31), en gros plan plus grand que nature (fig. 4), lorsqu'elle prend la parole pour répondre en ces termes au loup gémissant sur son sort :

Aujourd'hui, vous êtes seul et je ne le suis pas. Vous êtes malheureux et je ne le suis pas. Vous êtes fragile et je ne le suis plus. Vous m'avez dévorée hier. Je viens me promener avec vous aujourd'hui. C'est que j'aime profondément la forêt, l'odeur du sous-bois, le soupir des arbres, le vol fou des geais. Vous ne m'avez pas pris cela. J'ai des lendemains radieux. [...]

Nous croyez-vous seuls? Nous ne le sommes pas! Avec votre fauteuil, je pousse vos crocs et mes blessures. Mais tour de roue après tour de roue, je me prouve que rien n'est jamais perdu. Je vous rends visite parce que je suis là. Debout. Malgré vous. Je veux croire qu'il est possible de devenir grand sans devenir méchant. Et je prends soin de vous pour le croire toujours (30).

Je veux être assez forte pour pouvoir aimer.

Même vous (32). 
$<$ Image en attente de droits>

Fig. 4 - H. Meunier et R. Lejonc, Cœur de bois. @ Éditions Notari, 2016.

17 La dernière double page cadre le couple sur le chemin du retour, en plongée, à peine perceptible au cœur de la forêt, enserré dans l'entrelacs des branches brunes (32-33).

La déclaration d'Aurore vient éclairer rétrospectivement toute l'histoire, et jusqu'à son prénom répété vingt fois par le narrateur au fil de l'album, telle une incantation, prénom métaphore qui crée une trouée de lumière dans la forêt hivernale, transcende les «blessures » d'enfance et annonce "des lendemains radieux ». Peut-être Henri Meunier aura-t-il eu connaissance de l'interprétation ritualiste de Pierre Saintyves qui voyait justement dans la couleur rouge du conte celle de l'aurore ${ }^{21}$. Au premier abord, cette réécriture $\mathrm{du}$ "Petit Chaperon rouge " illustre un certain empowerment ${ }^{22} \mathrm{du}$ féminin : la magnification de la jeune femme et son initiative font d'elle un personnage central et moteur, alors que le vieux loup déchu, paralysé par l'âge et la maladie, ne joue plus qu'un rôle passif. Comme le conte source, l'album se clôt sur un dialogue, mais c'est le personnage féminin qui monopolise la parole, et qui a le dernier mot. On pourrait se contenter de voir, dans ce retournement, une revanche du féminin et la marque d'une justice immanente conforme à la règle selon laquelle, depuis la Poétique d'Aristote, dans une œuvre littéraire, le coupable doit être châtié - règle pourtant non respectée dans le conte de Perrault, contrairement à certaines versions populaires et à la réécriture des Grimm. Mais le propos d'Aurore porte un discours plus grave et plus sensible, scandé par la réitération du pronom de la première personne du singulier et par des formules volontaristes : "je veux ", «je me prouve que ». Ni infirmière, ni aide à domicile (les deux seuls visiteurs du vieillard), animée par une puissante pulsion de vie, elle se présente en femme "debout", capable de regarder le passé en face, sans oubli ni pardon. La suite (sequel) imaginée par Henri Meunier vise en effet à répondre à la question de Richard Saint-Gelais, que nous rappelions plus haut, sur le destin du personnage: «qu'arrivera-t-il ensuite?» Comment pourra vivre la victime d'un traumatisme? Le charme suranné du conte source, de ce récit trop entendu et trop bien connu que l'on aurait tort de croire poli par l'usage, ne saurait faire oublier la violence qui le sous-tend. Anne-Marie Garat ne trouve pas de mots assez forts pour le rappeler : " [...] il est horrible. Un cauchemar absolu », avec son " cocktail explosif de sexe et de mort [...] rapt, viol, inceste, pédophilie, cannibalisme $»^{23}$. Dans un album qui a fait date, Sarah Moon avait su traduire l'agression subie, en terminant son Petit Chaperon rouge ${ }^{24}$ par la photographie en plongée d'un lit dévasté, théâtre du viol. On pourrait imaginer que Meunier et Lejonc reprennent ici le fil du drame, pour montrer ce qu'il est advenu de la petite fille quelque trente-cinq ans plus tard.

La démarche d'Aurore illustre les thèses de Boris Cyrulnik sur la résilience, terme dont il rappelle l'origine dans son manifeste :

En physique, il définit l'aptitude d'un corps à conserver sa structure quelles que soient les pressions du milieu. Mais en latin, le verbe resilio ajoute une notion de ressaut, le fait de revenir en sautant ; peut-être rebondir après avoir subi le recul du coup ? ${ }^{25}$

Tout son travail de neuropsychiatre a consisté à se demander «comment certains enfants ont pu devenir des adultes épanouis malgré des conditions adverses» et si " une vie est encore possible après la blessure du traumatisme. Comment la relancer? À quel prix ?». Il explique que pour celui qui a connu « le fracas, qui a plus ou moins bien cicatrisé », la souffrance est inévitable et qu'il faut apprendre à la transformer, car 
"c'est autour d'une souffrance surmontée ou enfouie que s'organise la reprise du développement de la personnalité ${ }^{26}$. Certains créateurs d'albums ont mis en œuvre ce processus de résilience en remaniant des contes dans lesquels les personnages d'enfants sont maltraités, voire violentés : dans Un petit chaperon rouge de Claude Clément, illustré par Isabelle Forestier (Grasset Jeunesse, 2000), la fillette prostrée après l'agression, reprend pied grâce à l'aide d'une parole libératrice et du jugement qui officialise le crime du loup ; dans Le Coeur de Violette de Michel Piquemal, illustré par Nathalie Novi (La Martinière Jeunesse, 2000), réécriture de «Peau d'âne » dans laquelle l'inceste est consommé, la jeune fille dont le cœur s'était endurci revient sur le versant positif de la vie grâce à l'amour d'un jeune homme; dans nombre de reprises du « Petit Poucet ${ }^{27}$, les tourments de l'enfance sont surmontés lorsque le petit personnage trouve une raison de vivre qui lui « prouve que rien n'est jamais perdu», comme le dit Aurore elle-même.

Henri Meunier et Régis Lejonc parviennent à traduire ce processus complexe à travers les nuances du texte et la force des images. Si Aurore parait pleine d'allant dans toute la première partie de l'album, elle marque un temps d'arrêt lorsqu'elle arrive à destination, comme s'il lui fallait prendre courage : "Aurore respira profondément, immobile, puis s'avança vers la porte d'entrée » (16). Elle lance un salut joyeux à la cantonade et manifeste une sollicitude attentive pour préparer le goûter : ainsi, elle prévient tout risque de fausse route en plaçant la couronne sur l'assiette du vieillard, " afin de ne pas faire mystère de sa malice. Un roi qui s'étouffe n'est pas roi tout à fait " (20). Quand elle approche du fauteuil sur lequel il somnole, c'est « avec délicatesse [qu'] elle lui effleur[e] le genou» (21). Leurs premiers échanges relèvent d'«une conversation convenue au mot près »: «il s'enquit de la scolarité des petites, des voyages de son mari. Aurore s'inquiéta en retour de son état de santé» (23); mais ce vernis de normalisation se craquelle ensuite lorsque le loup tombe de sa chaise en déplorant : «J'étais fort autrefois », car Aurore dément : « Non. Non, vous n'avez jamais été fort. Vous étiez puissant. C'est autre chose » (24). Durant la promenade, alternent les propos de convention et ceux qui réfèrent au passé palpitant sous les apparences, jusqu'au moment qui précède la tirade finale d'Aurore, quand le loup en vient à se plaindre de sa solitude :

"Je n'ai que vous, fillette. Et je ne comprends pas pourquoi. Je ne comprends pas

vos attentions pour moi qui, naguère, vous ai dévorée toute crue. Je ne comprends pas votre pardon ».

"Je ne vous ai rien pardonné », souffla Aurore.

«Je suis désolé », bougonna le vieillard impotent.

"Je n'ai pas besoin d'excuses non plus», ajouta-t-elle en admirant la chute

hasardeuse d'un premier flocon (28).

La déclaration finale d'Aurore (30), confrontée à l'image de son beau «visage impassible » (31), masque pâle et déterminé, rappelle que le souvenir des souffrances reste intact, omniprésent : «Avec votre fauteuil, je pousse vos crocs et mes blessures ». Il ne s'agit pas d'oublier, mais de vivre avec, de dépasser les sentiments négatifs, d'apprivoiser la douleur et le bourreau lui-même, qui a perdu toute superbe et sa puissance passée. C'est grâce à ce tour de force qui convertit la haine et la rancune en amour qu'Aurore peut prendre sa revanche sur le malheur. Le ton sur lequel elle s'exprime révèle que le chemin choisi n'est pas le plus facile, ce que les images de Régis Lejonc rappellent à leur manière, car la gamme chromatique de l'album reste constamment sombre, avec de rares éclats plus pâles et quelques traits de rouge. On 
retient surtout la peinture d'une forêt obscure, inextricable, dans laquelle les chemins se perdent et où surgit soudain une bâtisse délabrée, à la silhouette menaçante. Une forêt qu'Henri Meunier évoque en termes funèbres avec ses «arbres décharnés » (14), "le squelette dansant des arbres » (26). La dernière image en double page (32-33) qui montre la nuit tombant sur le bois contraste avec les derniers mots d'Aurore : « Je veux être assez forte pour pouvoir aimer. Même vous » (32); elle donne la part belle à la sombre forêt des contes, celle qui suscite l'angoisse, dans laquelle le danger rôde et où l'on risque de se perdre.

Se sauver ou se perdre, cette ambiguïté peut se lire dès le titre de l'album, syntagme qui se prête à différentes interprétations. Il pourrait désigner métaphoriquement le lieu de l'action, le cœur de la forêt, au milieu du bois. Mais la proposition retenue mentionne un « cœur de bois » (et non « du » bois), et l'on peut penser à une autre métaphore, qui serait formée sur le modèle d'un «cœur de pierre ». Mais qui aurait ici un « cœur de bois », donc d'une essence plus propre à s'attendrir que la pierre ?28 Il se trouve que le «cœur de bois» est une expression qui existe, dans le domaine spécifique de la sylviculture: elle désigne le centre d'un tronc, la zone que l'on nomme scientifiquement duramen ${ }^{29}$, celle où le bois a durci, contrairement aux strates extérieures de l'aubier, plus tendres et plus friables. Le « cœur de bois » pourrait-il être une nouvelle métaphore pour désigner l'aboutissement du processus de résilience qui a permis à Aurore de surmonter son traumatisme, de s'endurcir suffisamment pour affronter son adversaire? Mais si l'on regarde de près l'étymologie du mot duramen, on observe qu'il est en réalité composé à partir du verbe durare, qui signifie «durer » et non « durcir ». Le cœur de bois, c'est la partie résistante du bois, celle qui a gagné en consistance au fil du temps et qui est devenue plus solide. La métaphore désignerait alors de façon positive la victoire d'Aurore sur elle-même, qui lui a permis de survivre au viol subi dans l'enfance, non seulement de continuer à vivre, mais de s'épanouir, de devenir « forte » (30), après avoir été « fragile » (28).

\section{Conclusion}

La lecture de la suite du «Petit Chaperon rouge » d'après Henri Meunier et Régis Lejonc ne laisse pas le lecteur indemne et l'on est en droit de se demander si cet album s'adresse réellement à des enfants. Spécialisées dans les livres d'art, les Éditions Notari ont bien ouvert un secteur de livres pour la jeunesse «choisis en fonction de leur valeur artistique et éducative $\aleph^{30}$. Cependant, la collection dans laquelle est placé Couur de bois s'adresse manifestement à un double public ${ }^{31}$. Experts ou novices, les lecteurs liront entre les lignes ou percevront de manière plus intuitive la portée d'une histoire qui ne relève pas du conte d'avertissement, comme le conte source. Il ne s'agit plus ici de prévenir, de mettre en garde, mais de guérir, de proposer des palliatifs pour réparer les blessures subies, de montrer que des lendemains meilleurs sont possibles. Les deux créateurs rejouent la partition du «Petit Chaperon rouge » à leur manière en tressant les fils des anciennes versions au gré d'allusions ou de références. Perrault opposait le conte - tissé d'archaïsmes propres à traduire la poésie rurale et l'esprit d'enfance - à sa moralité, qui invite "à retrouver dans le conte ce qu'aurait imaginé un conteur plus gaillard, averti des dangers auxquels on est exposé dans le milieu des lecteurs, et à lui attribuer des doubles sens $»^{32}$. La version des Grimm se voulait ouvertement éducative. Aujourd'hui, Henri Meunier et Régis Lejonc proposent une interprétation qui se fonde 
sur les tendances de la sensibilité contemporaine ${ }^{33}$, et ils se montrent capables d'en jouer sans peser. À travers le jeu du texte et des images, ils parviennent à relier les extrêmes : la trivialité du quotidien, l'horreur du crime, le bonheur des sensations, le vouloir vivre, le tout baigné dans le souvenir du conte qui enveloppe les images et confère à une histoire tristement ou banalement réaliste une aura merveilleuse.

\section{BIBLIOGRAPHIE}

\section{Sources}

CLEMENT, Claude, Un petit chaperon rouge, illustré par Isabelle Forestier, Paris, Grasset Jeunesse, 2000.

DANTE, La Divine comédie : L'Enfer, trad. par Jacqueline Risset, Paris, Flammarion, « GF bilingue », 1992.

FDIDA, Jean-Jacques, Le Petit Chaperon rouge ou La Petite Fille aux habits de fer-blanc, illustré par Régis Lejonc, Paris, Didier Jeunesse, 2010.

JOIRET, Patricia, Mina Je t'aime, illustré par Xavier Bruyere, Paris, L'École des loisirs/Bruxelles, Pastel, 1991.

MEUNIER, Henri, Cœur de bois, illustré par Régis Lejonc, Genève, Éditions Notari, 2016.

PERRAUlt, Charles, Contes, Paris, Le Livre de Poche classique, 1990.

-, Le Petit Chaperon rouge, illustré par Sarah Moon, Paris, Grasset \& Fasquelle, 1983.

PIQUEMAL, Michel, Le Cœur de Violette, illustré par Nathalie Novi, La Martinière Jeunesse, 2000.

\section{Travaux}

BECKETT, Sandra L., Recycling Red Riding Hood, New York/London, Routledge, 2002.

-, Crossover Fiction : Global and Historical Perspectives, New York, Taylor \& Francis, 2008.

-, Crossover Picturebooks : A Genre for All Ages, New York, Routledge, 2011.

BLOCH, Jeanne, «Le héros animal dans les contes de fées de Mme d'Aulnoy : Le Prince Marcassin, Serpentin Vert, La Chatte blanche, La Biche au bois ", Dix-huitième siècle, 42 (2010/1), p. 119-138 (<https://www.cairn.info/revue-dix-huitieme-siecle-2010-1-page-119.htm>).

CALVÈs, Anne-Emmanuèle, « "Empowerment " : généalogie d'un concept clé du discours contemporain sur le développement », Revue Tiers Monde, 200 (2009/4), p. 735-749.

CONNAN-PINTADO, Christiane, « Conjurer malveillance et maltraitance dans l'album contemporain pour la jeunesse. L'exemple du Petit Poucet », in Malveillance/maltraitance de l'enfant dans les récits pour jeune public, éd. par Kveta Kunesova, Thierry Charnay, Université Hradec Kralové, «Gaudeamus », 2017, p. 23-31. 
-, « Avant-propos ", in L'épanchement du conte dans la littérature, éd. par Christiane ConnanPintado, Pascale Auraix-Jonchière, Gilles Béhotéguy, Bordeaux, Presses universitaires de Bordeaux, 2018, p. 5-12 (« Modernités », 43).

CYRULNIK, Boris, « Manifeste pour la résilience », Spirale, 18 (2001/2), p. 77-82 (< https://

www.cairn.info/revue-spirale-2001-2-page-77.htm<).

DELARUE, Paul, TENÈZE, Marie-Louise, Le conte populaire français, Paris, Maisonneuve et Laroze, 1997 [1957].

ERNY, Pierre, Sur les traces du Petit Chaperon rouge : un itinéraire dans la forêt des contes, Paris, L'Harmattan, 2003.

GARAT, Anne-Marie, Une faim de loup. Lecture du Petit Chaperon rouge, Arles, Actes Sud, 2004.

GEFEN, Alexandre, Réparer le monde. La littérature française face au XXI ${ }^{e}$ siècle, Paris, José Corti, 2017.

GENETTE, Gérard, Palimpsestes. La littérature au second degré, Paris, Éditions du Seuil, 1982.

LA GENARDIÈRE, Claude de, Encore un conte? Le Petit Chaperon rouge à l'usage des adultes, Paris, L'Harmattan, 1996.

SAINT-GELAIS, Richard, Fictions transfuges. La transfictionnalité et ses enjeux, Paris, Éditions du Seuil, 2011.

SAINTYVES, Pierre, Les contes de Perrault et les récits parallèles, leurs origines, coutumes primitives et liturgies populaires, Paris, Slatkine, 1990 [Nourry, 1923].

SERMAIN, Jean-Paul, Le conte de fées, du classicisme aux Lumières, Paris, Desjonquères, 2007.

VERDIER, Yvonne, "Grand-mère si vous saviez... : Le Petit Chaperon rouge dans la tradition orale ", in Les Cahiers de littérature orale, IV (1978) (<http://expositions.bnf.fr/contes/cles/verdier.htm>).

\section{NOTES}

1. Dante, La Divine comédie, chant I, v. 1-6 (éd. cit., p. 24 sq.) : « Nel mezzo del cammin di nostra $\mathrm{vita} / \mathrm{mi}$ ritrovai per una selva oscura,/ché la diritta via era smarrita.//Ahi quanto a dir qual era è cosa dura/esta selva selvaggia e aspra e forte/che nel pensier rinova la paura ! ».

2. A.-M. Garat, Une faim de loup, p. 16.

3. Outre celle d'A.-M. Garat, on peut citer les monographies de C. de La Genardière, Encore un conte?; de S. L. Beckett, Recycling Red Riding Hood et de P. Erny, Sur les traces du Petit Chaperon rouge.

4. La famille ogre (L'Atelier du poisson soluble, 2004), minuscule album au format d'un jeu de cartes et rangé dans un étui, décline les membres d'une famille en faisant référence à différents ogres des contes. Une autre fois (Éditions du Rouergue, 2005) est à la fois une réécriture du « Petit Poucet ", un conte en randonnée et un mélange de contes puisque les enfants perdus rencontrent successivement les autres personnages de Perrault. Cent Grillons (Éditions du Rouergue, 2013) est un recueil de réécritures de contes fondées sur des jeux de mots, comme l'indique son titre.

5. J.-J. Fdida, Le Petit Chaperon rouge ou La Petite Fille aux habits de fer-blanc, illustré par R. Lejonc.

6. L'album n'étant pas paginé, nous attribuons les numéros de 1 à 36 de la première à la quatrième de couverture. Pour les images, voir: <http://www.editionsnotari.ch/collections/ loiseau-sur-le-rhino/les-herons/coeur-de-bois.html>.

7. A. Gefen, Réparer le monde.

8. Voir notre « Avant-propos » à L'épanchement du conte dans la littérature, p. 5-12. 
9. Henri Meunier puise à toutes les sources, littéraires et populaires et il connaît manifestement la version nivernaise, «Conte de la mère grand ", rapportée par Paul Delarue (Le Conte populaire français, p. 373 sq.), dans laquelle c'est le «bzou » qui pose cette question. Sa reprise ne manque pas ici de sel, car il écrit qu'Aurore la formule d'un ton « candide».

10. G. Genette, Palimpsestes, p. 222 sqq.

11. R. Saint-Gelais, Fictions transfuges, p. 71.

12. Ibid., p. 76.

13. Y. Verdier « Grand-mère si vous saviez... ».

14. Voir le chapitre intitulé «Dans l'entre-deux-mères », in C. de La Genardière, Encore un conte?, p. 81 sqq.

15. J. Bloch, «Le héros animal dans les contes de fées de Mme d'Aulnoy », p. 120.

16. Nous empruntons ce terme théorisé et illustré par S. L. Beckett dans Crossover Picturebooks et Crossover Fiction pour désigner les œuvres adressées à un double lectorat.

17. Ch. Perrault, Contes, p. 196 sq.

18. J.-P. Fdida, Le Petit Chaperon rouge ou La Petite Fille aux habits de fer-blanc, ill. R. Lejonc.

19. P. Joiret, Mina je t'aime, illustré par X. Bruyère. Dans cette réécriture-réappropriation du conte, on découvre à la dernière page que la jeune Carmina, dont l'allure sexy affole les garçons du village, les appâte en réalité pour les conduire jusqu'à sa grand-mère louve.

20. À une réserve près : le gros plan révèle que tout en reprenant les traits et l'expression de l'actrice, Régis Lejonc colore en bleu les yeux d'Aurore.

21. P. Saintyves, Les contes de Perrault et les récits parallèles, p. 215 sqq.

22. A.-E. Calvès, “"Empowerment”", p. 735: «Le terme “empowerment”, qui signifie littéralement "renforcer ou acquérir du pouvoir" est utilisé abondamment [...] dans le discours féministe et radical des années $1980 »$.

23. A.-M. Garat, Une faim de loup, p. 64 sq.

24. Ch. Perrault, Le Petit Chaperon rouge, illustré par Sarah Moon.

25. B. Cyrulnik, « Manifeste pour la résilience », §9.

26. Ibid., § 16 à 20 .

27. Ch. Connan-Pintado, "Conjurer malveillance et maltraitance dans l'album contemporain pour la jeunesse ».

28. La formule ferait ainsi écho à celle de Perrault dans "La Barbe bleue » : "Elle aurait attendri un rocher, belle et affligée comme elle était ; mais la Barbe bleue avait le cœur plus dur qu'un rocher » (Ch. Perrault, Contes, p. 209).

29. Voir le dictionnaire Littré (<https://www.littre.org/definition/duramen>), consulté en ligne le $24 / 06 / 2018$.

30. Voir le site de l'éditeur (<http://www.editionsnotari.ch/a-propos.html>), consulté en ligne le 25/06/2018.

31. La collection porte un titre métaphorique qui désigne le double destinataire, «L'oiseau sur le rhino ». Il est précisé dans le colophon que Cour de bois s'inscrit dans une section manifestement crossover: «Les hérons (pour les plus grands et leurs parents)».

32. J.-P. Sermain, Le conte de fées, du classicisme aux Lumières, p. 96.

33. Voir l'ouvrage d'A. Gefen. 


\section{RÉSUMÉS}

L'album iconotextuel Cour de bois (2016) se présente comme une continuation du «Petit Chaperon rouge " située à l'époque contemporaine. Empruntant aux versions littéraires et populaires du conte, il relate une visite de la victime à son bourreau, quelques décennies plus tard, et inscrit son histoire de résilience dans le courant littéraire contemporain qui se donne pour objectif de « réparer les vivants ». Les images qui dialoguent avec le texte tissent un réseau de ramifications étrangement inquiétantes en réactivant les séductions et les dangers de la forêt profonde et des passions humaines. L'article prête attention à la présence latente du conte source, aux atouts de l'album pour la mettre en valeur et à la portée idéologique de l'interprétation.

The iconotextual picturebook Coeur de bois (2017) is a continuation (or sequel) of the "Little Red Riding Hood" story set in the contemporary era. Borrowing from the literary and popular versions of the tale, it recounts a visit paid by the victim to her tormentor a few decades after the abuse, and places this story of resilience in a contemporary movement that seeks to "heal the living" through literature. The images that interact with the text weave a network of strangely disturbing ramifications by reactivating the seductions and dangers of the deep forest and human passions. The article notably focuses on the latent presence of the source text and on the picturebook's strategies to draw attention to it, and discusses the ideological implications of this new interpretation of the old tale.

\section{AUTEUR}

\section{CHRISTIANE CONNAN-PINTADO}

Université Bordeaux Montaigne 